\title{
Exciton Polaritons Reveal "Hidden" Populations in Functionalized Pentacene Films
}

Jonathan D. B. Van Schenck ${ }^{1}$, Winston T. Goldthwaite ${ }^{1}$, Richard Puro ${ }^{1}$, John E. Anthony $^{2}$, and Oksana Ostroverkhova*1

${ }^{1}$ Department of Physics, Oregon State University, Corvallis, OR 97331

${ }^{2}$ Center for Applied Energy Research, University of Kentucky, Lexington, KY 40511

\section{Contents}

\begin{tabular}{ll}
\hline S1 Average Molecular Spacing and Molecular Densities & S1
\end{tabular}

\begin{tabular}{ll}
\hline S2 X-Ray Diffraction & S3
\end{tabular}

$\begin{array}{ll}\text { S3 Thermal Annealing } & \text { S4 }\end{array}$

$\begin{array}{ll}\text { S4 Ellipsometry } & \text { S7 }\end{array}$

\begin{tabular}{ll}
\hline S5 Spectral analysis & S8
\end{tabular}

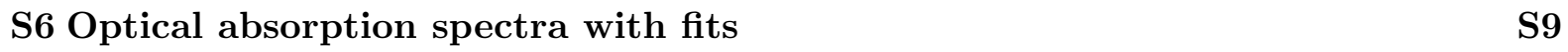

\begin{tabular}{|l|l|}
\hline S7 Photoluminescence spectra and lifetimes & S13
\end{tabular}

\begin{tabular}{|l|l|}
\hline S8 Polariton properties & S14
\end{tabular}

\begin{tabular}{lr}
\hline S9 Density functional theory & S19
\end{tabular}

\section{List of Figures}

S1 X-ray diffraction data for films under study. . . . . . . . . . . . . . . . . . . S4

S2 Evolution of optical absorption spectra of a pristine TIPS-Pn film during the thermal annealing process. . . . . . . . . . ..... S6

S3 Absorption spectra for the pristine TIPS-Pn film in a cavity before and after

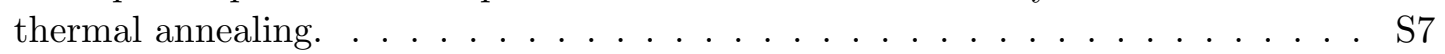

S4 $\quad$ Absorption spectrum with fits for TIPS-Pn in dilute solution. . . . . . . . . . . . S9

S5 Absorption spectrum with fits for TIPS-Pn:PMMA film $F_{2}^{(B)}$. . . . . . . . . . S10

S6 $\quad$ Absorption spectrum with fits for TIPS-Pn:PMMA film $F_{13}^{(B)}$. . . . . . . . . . S10

S7 Absorption spectrum with fits for TIPS-Pn:PMMA film $F_{1}^{(B)}$. . . . . . . . . S11

S8 $\quad$ Absorption spectrum with fits for pristine TIPS-Pn film $F^{(P)}$. . . . . . . . . . . S12

S9 $\quad$ Absorption spectrum with fits for pristine TIPS-Pn film $A^{(P)}$. . . . . . . . . . . S12

S10 $\quad$ PL spectra for selected films and solution. . . . . . . . . . . . . . . . S13

S11 PL lifetime decays for selected films and solution. . . . . . . . . . . . . . . . . . S13

S12 Cavity data for the mixed-phase blend $F_{1.3}^{(B)} . \ldots \ldots \ldots$. . . . . . . S16

S13 Comparison of the cavity data for TIPS-Pn- and diF TES-ADT-based films. . . S17 
S14 Cavity data for TIPS-Tc amorphous film. . . . . . . . . . . . . . . . . . S18

S15 Scaling of the interaction strength $2 V_{0 m}$ with the oscillator strength $f_{0 m}$ in TIPSTc cavities . . . . . . . . . . . . . . . . . . . . . . . . . . .

\section{List of Tables}

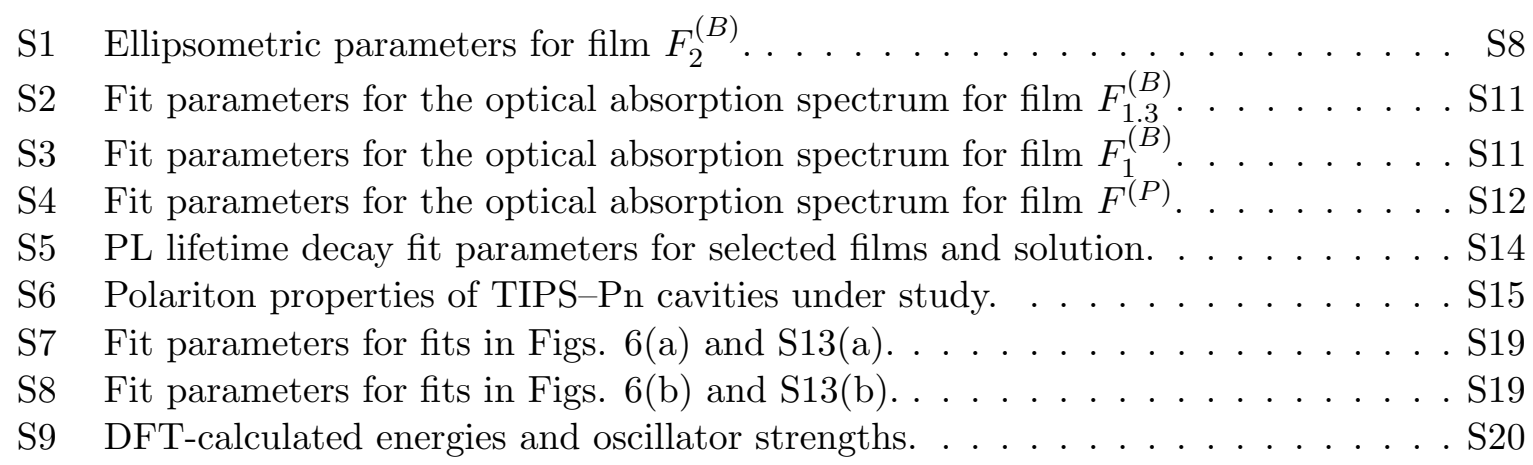

\section{S1 Average Molecular Spacing and Molecular Densities}

For blended films such as $F_{2}^{(B)}$, the average molecular spacings were controlled by varying the relative concentrations of TIPS-Pn and PMMA molecules in the solutions used for spincasting these films, in keeping with our previous publications [1, 2]. By assuming that the guest molecules are isotropically distributed throughout the host polymer, each guest molecule has a sphere of polymer surrounding it which contains no other guest molecules, the volumes of which are:

$$
\mathcal{V}_{G}=\frac{4}{3} \pi r^{3}
$$

The radii $(r)$ of these spheres are expected to have some distribution, where the average radius is $\langle r\rangle$, which defines also the average volume:

$$
\left\langle\mathcal{V}_{G}\right\rangle \equiv \frac{4}{3} \pi\langle r\rangle^{3}
$$

The average spacing between molecules can then be defined as:

$$
d \equiv 2\langle r\rangle
$$

These guest-host blended films are deposited by spin-casting a solution which contains both the guest and host molecules dissolved within. The $f$-number of the solution for deposition is defined as:

$$
f \equiv \frac{C_{H}}{C_{G}}
$$

where $C_{G}\left(C_{H}\right)$ is the molar concentration of the guest (host) molecule in the solution. If it is assumed that the relative concentrations of the guest and host molecules in the solution is the same as the relative number densities of the guest and host molecules in the films, then the $f$-number should also match the ratio of the two number densities:

$$
f=\frac{n_{H}}{n_{G}}=\left\langle\mathcal{V}_{G}\right\rangle n_{H}
$$

where $\left\langle\mathcal{V}_{G}\right\rangle=1 / n_{G}$ follows from the assumption of isotropic distribution. Finally, if the number density of the host polymer is assumed to be approximately the same as the number density of 
the bulk host polymer (i.e. without any guest molecules), then the host number density can be expressed in terms of the bulk host mass density:

$$
n_{H} \approx \frac{N_{A} \cdot \rho_{H}^{(\text {bulk })}}{M_{H}}
$$

where $N_{A}$ is Avogadro number, $\rho_{H}^{(\text {bulk) }}$ is the bulk mass density of the polymer host (i.e. without any guest molecules) and $M_{H}$ is the molar mass of the host. Combining Eq. S2 and Eq. S5, one can derive:

$$
\begin{aligned}
f & =n_{H} \frac{4}{3} \pi\langle r\rangle^{3} \\
& =n_{H} \frac{4}{3} \pi\left(\frac{d}{2}\right)^{3} \\
& \approx \frac{N_{A} \cdot \rho_{H}^{\text {(bulk })}}{M_{H}} \cdot \frac{4}{3} \pi\left(\frac{d}{2}\right)^{3}
\end{aligned}
$$

Which rearranging to solve for $d$ yields:

$$
d=\sqrt[3]{\frac{6 M_{H}}{\pi N_{A} \rho_{H}^{(\mathrm{bulk})}} \cdot f}
$$

which, in the case of PMMA as the host polymer with the bulk values $M_{\text {PMMA }}=100 \mathrm{~g} / \mathrm{mol}$ and $\rho_{\text {PMMA }}^{\text {(bulk) }}=1.18 \mathrm{~g} / \mathrm{cm}^{3}$, yield:

$$
d^{(\text {in PMMA })} \approx(0.644 n m) \sqrt[3]{f}
$$

And so, using this and Eq. S4, the average molecular spacing of guest molecules in a PMMA polymer matrix can be calculated from the solution concentration values.

When the number of guest molecules relative to the number of host molecules becomes large $(f>1)$, the blended films described above are no longer dominated by the host film formation dynamics, but rather become dominated by the guest film formation dynamics, with the limiting case being a pristine film of only guest molecules $(f \rightarrow \infty)$. In these cases, the above derivation is no longer valid, as guest molecules will have a strong propensity to aggregate rather than being isotropically distributed through the polymer. The average molecular spacing can thus be recovered by using the geometry of the aggregates.

For crystalline aggregates, x-ray crystallography can find the intermolecular orientations and distance allowing for the construction an "average molecular distance" which is comparable with that constructed above. The approach is to assume that each molecule is at the center of a region of space (containing only this molecule), and then construct $d$ as twice the characteristic radius of this region. However, many organic crystals form a triclinic crystalline structure, where the lengths between adjacent molecules are highly anisotropic. So, rather than a sphere to model the volume inhabited by a single molecule, an ellipsoid is preferred. Therefore,

$$
\mathcal{V}_{G} \equiv \frac{4}{3} \pi A B C
$$

where $A, B, C$ are the axes of the ellipsoid centered on the particular molecule. By taking an average of these volumes (in the case that $Z>1$ ), the average molecular distance can then be constructed by equating the volume of the ellipsoid with a theoretical sphere:

$$
\left\langle\mathcal{V}_{G}\right\rangle=\frac{4}{3} \pi\left(\frac{d}{2}\right)^{3}
$$




$$
\Rightarrow d=\sqrt[3]{\frac{6}{\pi}\left\langle\mathcal{V}_{G}\right\rangle}
$$

In the special case where $Z=1$ and the crystal is approximately orthorhombic $\left(\alpha, \beta, \gamma \sim 90^{\circ}\right)$, then the values of $A, B, C$ are approximately half the crystal lattice spacings $a, b, c$, and so $d$ takes the reduced form:

$$
\Rightarrow d^{(Z=1, \text { Ortho })} \approx \sqrt[3]{a b c}
$$

In this case, the average molecular spacing is a geometric average of the lattice spacings, with $a=0.75650 \mathrm{~nm}, b=0.77500 \mathrm{~nm}, c=1.6835 \mathrm{~nm} 3$.

Finally for the pristine amorphous films, the volume was calculated from the thickness contraction experienced by films during the annealing process used for fabrication of amorphous films $\left(\mathcal{V}_{A^{(P)}}=(0.75 \pm 0.15) \mathcal{V}_{F^{(P)}}\right.$, see Figs. S2 and S3). In particular, we observe a clear blue shift of cavity resonance (from below $1.6 \mathrm{eV}$ to above $2.4 \mathrm{eV}$ in Fig. S3) upon the transition from the crystalline to the amorphous phase (obtained by monitoring the optical properties of the cavity in-situ during a thermal treatment to induce the transition, Fig. S2, as discussed in Section S3). The wavelength of the cavity resonance is primarily dependent upon the film thickness and the refractive index. The change in the refractive index between $F^{(P)}$ and $A^{(P)}$ films (see, for example, Table S6) is small and so it contributes little to this difference, and therefore the film thickness is the main factor. This implies that upon the transition from the crystalline (predominantly (001) orientation, with a relatively large c-axis lattice spacing of 1.7 $\mathrm{nm}$ ), to the amorphous phase, the film collapses so that the same initial number of molecules is now packed into a smaller space (yielding a smaller film thickness). Given the change in cavity resonance wavelength, we estimate that the film thickness would need to decrease to a $0.75 \pm 0.15$ fraction of the original thickness. We hypothesize that this out-of-plane change is the dominant change, and the in-plane molecular rearrangements for the closely-spaced molecules in the $a-b$ plane of the crystallites are less significant. These considerations translate into the change in volume of the film be approximately equal to the change in the thickness, so we estimate $\mathcal{V}_{A^{(P)}}=(0.75 \pm 0.15) \mathcal{V}_{F^{(P)}}$. The approximate nature of this calculation is reflected in the large error bars which are used with this estimate.

\section{S2 X-Ray Diffraction}

In order to confirm the crystallinity of $F^{(P)}$ films, and the effect of thermal annealing on crystallinity, we utilized X-Ray Diffraction measured using a Bruker D8 Discover diffractometer. Scattering intensity was measured for $2 \theta$ values between $3^{\circ}$ and $30^{\circ}$, with the upper bound chosen to be below the (111) scattering resonance of bulk silver. Figure S1(a) shows the scattering intensity from a pristine film of TIPS-Pn before and after thermal annealing. Both films have a low intensity background characteristic of films deposited on a glass-silver substrate (c.f. [1]).

The pre-annealed film shows crystalline resonances at $2 \theta=5.3^{\circ}, 10.7^{\circ}, 16.0^{\circ}$ corresponding to the $(00 l)$ (for $l=1,2,3)$ orientations of TIPS-Pn as well as an additional resonance at $2 \theta=12.8^{\circ}$ corresponding to the (011) orientation [3]. Similar crystalline orientations for pristine TIPS-Pn films have been previously observed in the literature, for example when deposited onto $\mathrm{CaF}_{2}$ substrates followed by solvent vapor annealing in Ref. [4].

The post-annealed film shows no crystalline resonances, confirming that our thermal annealing process effectively produces an amorphous film.

In order to correlate the appearance of the red-shifted absorption band (centered at 1.78 $\mathrm{eV}$ ) in more concentrated blends (as compared to $1.91 \mathrm{eV}$ in dilute blends) with the crystalline structure of films, we performed grazing-incidence X-ray diffraction experiments on select films under study. Figure S1(b) shows that no crystalline order is present in a dilute TIPS-Pn:PMMA blend $F_{2}^{(B)}$ (characterized by absorption spectra similar to those of dilute solutions, Fig. 2 in the main text), whereas more concentrated blends (such as a mixed-phase film $F_{1.3}^{(B)}$, exhibiting 
a $1.78 \mathrm{eV}$ absorption shoulder, in addition to the spectral features observed in dilute blends, Fig. S6) show the presence of crystallites, with the dominant (001) feature similar to that in pristine films $F^{(P)}$.
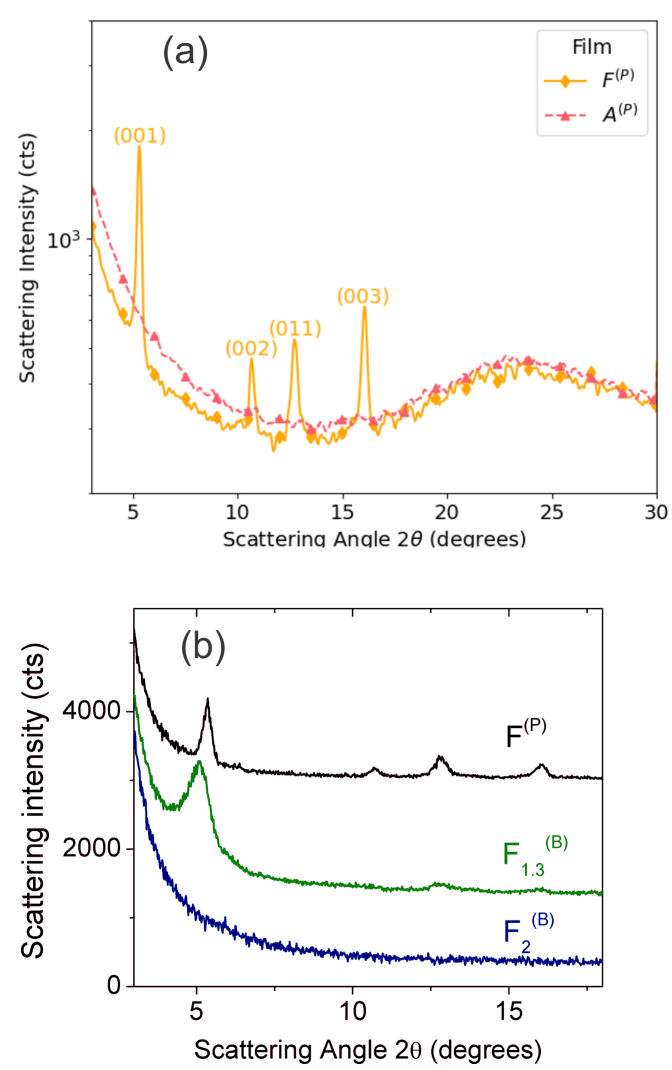

Figure S1: (a) XRD scattering intensity for a pristine film of TIPS-Pn on a silver substrate before $\left(F^{(P)}\right)$ and after $\left(A^{(P)}\right)$ annealing. Both films show the amorphous background signal characteristic of silver-on-glass substrate[1], however the $F^{(P)}$ sample also has a peak structure, revealing that both the $(00 l)(l=1,2,3)$ and (011) crystalline orientations are present in this film. These results are consistent with others works classifying "crystalline" and "amorphous" phases of TIPS-Pn, e.g. Ref. [4]. (b) GIXD intensity for a pristine crystalline film $F^{(P)}$, mixed-phase TIPS-Pn:PMMA film 1.3, and a dilute TIPS-Pn:PMMA blend 2. No crystalline features are observed in the dilute blend that has absorption spectra close to those of the dilute solutions (with the $0-0$ transition at $\sim 1.9 \mathrm{eV}$, Fig. 2 in the main text). In contrast, the presence of the crystalline phase is detected in the mixed-phase film that exhibits an absorption feature centered at $\sim 1.8 \mathrm{eV}$, in addition to that at $\sim 1.9 \mathrm{eV}$ (Fig. S6), which is similar to that in pristine crystalline films $F^{(P)}$ (Fig. 2 in the main text).

\section{S3 Thermal Annealing}

The pristine TIPS-Pn films deposited onto PFBT-treated silver substrates (as described in Sec. 2.1 of the main text) form a poly-crystalline phase upon deposition. This is evidenced both by the absorption spectrum of such films, which show a clear crystalline signature (see Fig. 2 in the main text, which exhibits a $\sim 700 \mathrm{~nm}$ red-edge absorption peak as the "crystalline" absorbance spectrum in, for example, Figure 1.A of Ref. [4]) and the XRD spectra from these films (see Sec. S2 and Fig. S1.

To force the pristine TIPS-Pn films into an amorphous phase, we employ thermal annealing, holding a sample at $250{ }^{\circ} \mathrm{C}$ on a hot plate. During annealing, films are exposed both to oxygen 
and light, which places these films at risk of both thermal- and photo-degradation. To study the effect of thermal annealing on these films, we monitored the reflectance in situ of $F^{(P)}$ samples (on silver-glass substrates) during a $10 \mathrm{~min}$ annealing process, which left the films almost entirely degraded. Figure S2 shows a summary of this reflectance data, which we discuss next.

Figure S2 shows the $1-R$ spectra (absolute in (a) and vertically shifted in (c) for clarity) from a $F^{(P)}$ film during the annealing process. During the first 2 seconds of the annealing process, the film is entirely poly-crystalline. This is evidenced by the prominent crystalline $(\sim 1.8 \mathrm{eV})$ absorption peak (labeled as $\left.E_{00}^{(\text {Crys. })}\right)$ and a lack of an amorphous ( 1.9 eV) absorption peak (labeled as $\left.E_{00}^{(A m o r p h .)}\right)$. Over the next few seconds, the film's background reflectance drops $(1-R$ increases) as the crystalline phase destabilizes causing the surface roughness to drastically increase. The film persists in this "rough surface" state until $t \approx 10 \mathrm{sec}$, at which point the film rapidly converts into an amorphous phase, evidenced by the rise of the amorphous $(\sim 1.9 \mathrm{eV})$ absorption peak and the rise of the background reflectance (drop in $1-R$ ). The amorphous phase molecules then begins to slowly degrade, leading to a gradual decrease in the $1-R$ at the amorphous absorption peak maximum. During this period $t=30-600 \mathrm{sec}$, there is a slight recovery of the poly-crystalline absorption peak, suggesting that the film partially re-crystallizes even as it degrades. Additionally, the overall $1-R$ increases again, due to internal scattering from degraded TIPS-Pn molecules. 

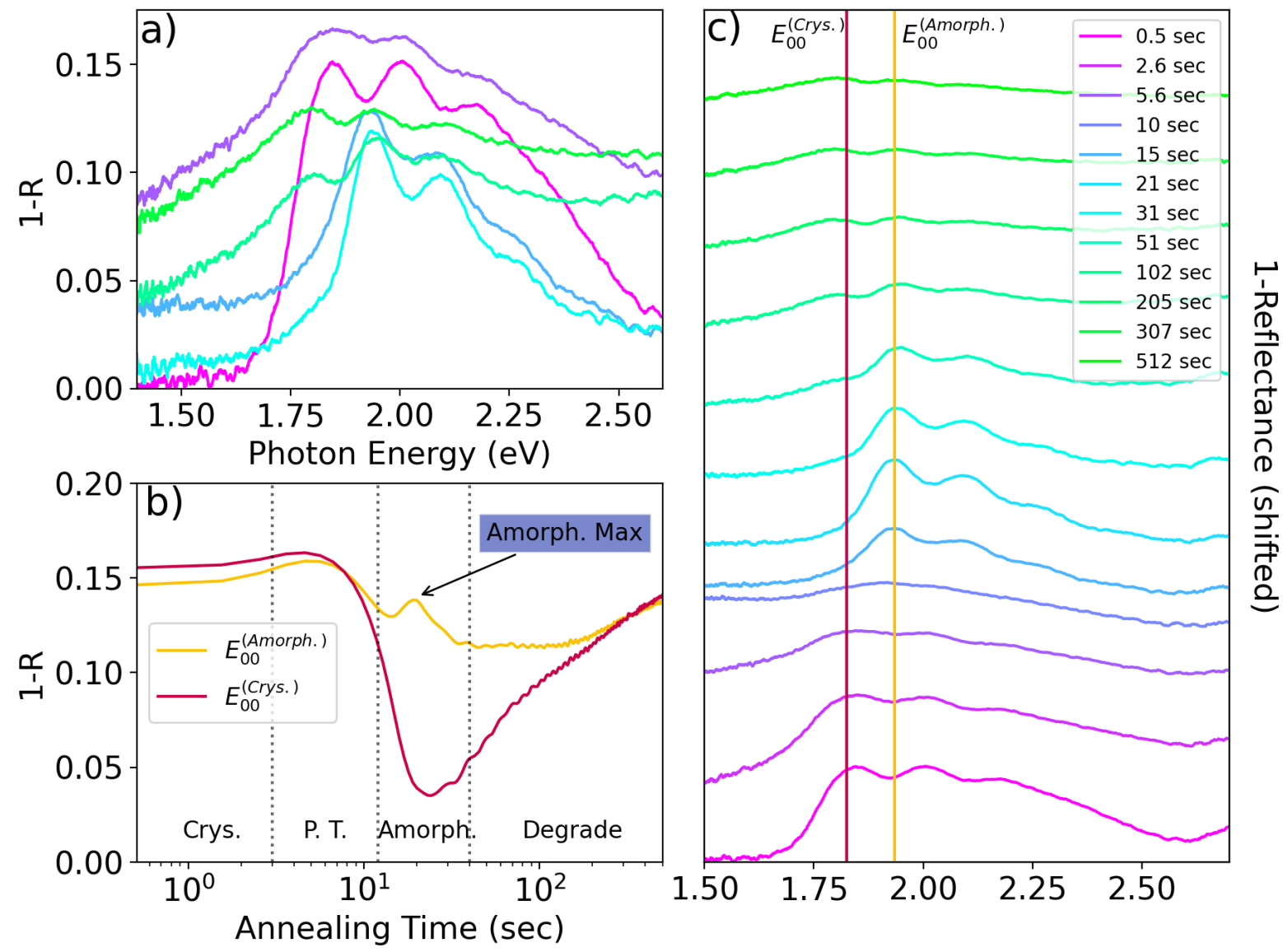

Figure S2: (a) Absorption $(1-R)$ of a pristine TIPS-Pn "bare film" at various times during thermal annealing, showing the transformation from a "crystalline" (main absorption peak at $\sim 1.8 \mathrm{eV}$ ) to an "amorphous" (main absorption peak at $\sim 1.9 \mathrm{eV}$ ) phase. (b) The absorption $(1-R)$ at the amorphous 0-0 transition energy $\left(E_{00}^{(\text {Amorph } .)}\right)$ and crystalline transition energy $\left(E_{00}^{(\text {Crys. })}\right)$, as a function of annealing time. Vertical dotted lines show demarcations for the four epochs of the annealing process: the crystalline phase ("Crys."), the phase transition ("P.T."), the amorphous phase ("Amorph.") and degradation ("Degrade"). Labeled ( $t \approx 20 \mathrm{sec}$ ) is the maximum of the amorphous phase exciton absorption time, representing the ideal annealing time to fully convert the film's phase with minimal degradation of the TIPS-Pn molecules. (c) The same absorption spectra from (a) but vertically shifted for clarity.

Figure S2(b) shows the $1-R$ spectrum at the wavelengths of the crystalline and amorphous absorption maxima as a function of annealing time. There are four temporal regions of the annealing process: (1) the crystalline phase (labeled "Crys."), followed by (2) a slight rise in the $1-R$ spectrum at both wavelengths (corresponding to the increasing of surface roughness) as the film begins to transition phases (labeled "P. T."); (3) a sudden rise in the amorphous $1-R$, as the crystalline $1-R$ drops rapidly, demonstrating that the film has entered an amorphous phase (labeled "Amorph."). The amorphous $1-R$ peaks at $t \approx 20 \mathrm{sec}$, before slowly dropping, corresponding to the molecules in the amorphous phase degrading. Finally, the overall $1-R$ spectrum rises as the molecules continue to degrade (labeled "Degrade") (4).

As a result of this analysis, to force crystalline films into the amorphous phase, in films $A^{(P)}$ discussed in the main text we used annealing for $20 \mathrm{sec}$ to maximize the number of molecules which form an amorphous phase, while minimizing the number which have thermally degraded. We note that although the annealing temperature used to creating these amorphous films is close to the decomposition temperature for TIPS-Pn, which likely contributes to the decay of 
absorption signal for long annealing times, the 20-second exposure is likely too short for the TIPS-Pn film to fully thermalize. This observation, together with the strong optical absorption signal from TIPS-Pn molecules which persists after annealing, demonstrates that decomposition of TIPS-Pn molecules play only a minor role in our chosen annealing process.

When the thermal annealing process is performed on microcavities, the cavity thickness dramatically decreases as can be seen from the shift in the cavity resonance in Fig. S3. This resonance shift was used to estimate a change in the molecular density in amorphous films $A^{(P)}$ as compared to crystalline films $F^{(P)}$ as described in the main text.

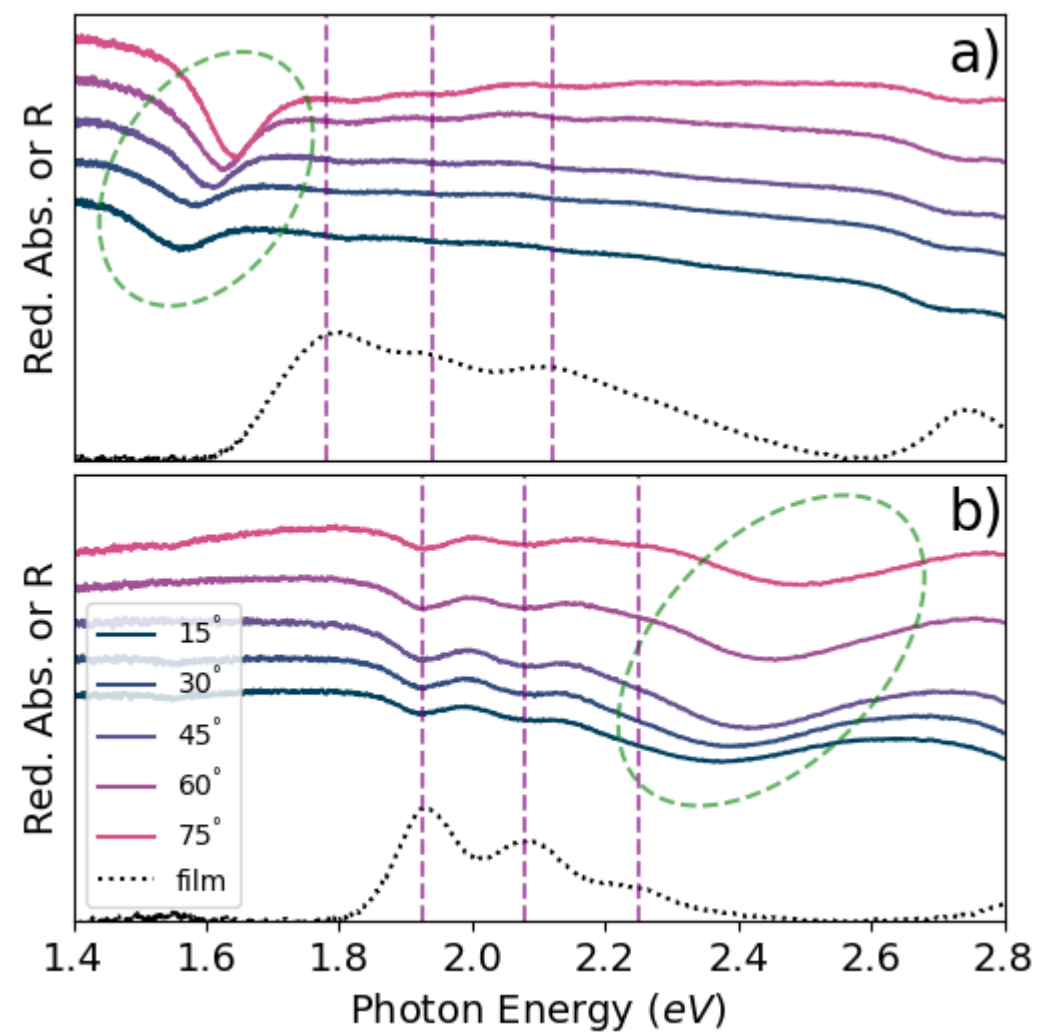

Figure S3: Reflectance spectra (vertically shifted for clarity) at several angles of incidence for a pristine TIPS-Pn optical cavity before (a) and after (b) thermal annealing. Also shown is the reduced absorption spectra from each film (dotted) and vertical dashed lines highlight the lowest three exciton energies. In both plots, the main feature in the reflectance spectra are emphasized by encircling. Because of the low reflectance of this feature and its blue-shifting dispersion, it is expected to have to have high photonic character. Since the features are separated by nearly $1 \mathrm{eV}$ between (a) and (b), the annealing process must drastically increase the photonic energy of a given cavity, which we attribute to a shrinking cavity thickness upon annealing.

\section{S4 Ellipsometry}

The index of refraction for TIPS-Pn:PMMA $(d=2 \mathrm{~nm}$ average molecular spacing $)$ film $F_{2}^{(B)}$ was extracted using spectroscopic ellipsometry on a J.A. Woollam Variable Angle Spectroscopic Ellipsometer. The index was modeled in two parts, a background and absorptive piece.

$$
n(\lambda)=n_{b g}(\lambda)+n_{a b s}(\lambda)
$$

The background index fit with a Cauchy model:

$$
n_{b g}(\lambda)=C_{0}+\frac{C_{1}}{\lambda^{2}}
$$


where $\lambda$ is the wavelength (in $\mu m$ ), and the absorptive index was fit using a Lorentz model:

$$
\left\{\begin{array}{l}
n_{a b s}(\lambda)=\sqrt{\epsilon_{r}(\hbar \omega=h c / \lambda)} \\
\epsilon_{r}(\hbar \omega)=\sum_{i=1}^{5} \frac{A_{i} E_{i} G_{i}}{E_{i}^{2}-\hbar \omega^{2}-i \hbar \omega G_{i}}
\end{array}\right.
$$

where $\hbar \omega$ is the photon energy, $A_{i}$ controls the oscillator strength of the $i^{\text {th }}$ transition, $E_{i}$ is the energy of the $i^{t h}$ transition in $e V$ and $G_{i}$ is the width of the $i^{t h}$ in $e V$. Table S1 gives the values of all fit parameters.

\begin{tabular}{c|c|c|c}
\multicolumn{2}{c|}{$C_{0}$} & \multicolumn{2}{c}{$C_{1}$} \\
\hline \multicolumn{2}{c|}{1.109} & \multicolumn{2}{|c}{$0.00733 \mu m^{2}$} \\
\hline \hline Index & $A_{i}$ & $E_{i}$ & $G_{i}$ \\
1 & 0.1724 & $1.916 \mathrm{eV}$ & $0.1302 \mathrm{eV}$ \\
2 & 0.07799 & $2.090 \mathrm{eV}$ & $0.08708 \mathrm{eV}$ \\
3 & 0.02334 & $2.254 \mathrm{eV}$ & $0.09987 \mathrm{eV}$ \\
4 & 0.01854 & $2.828 \mathrm{eV}$ & $0.03013 \mathrm{eV}$ \\
5 & 0.01064 & $2.993 \mathrm{eV}$ & $0.05279 \mathrm{eV}$
\end{tabular}

Table S1: Ellipsometric model parameters for $F_{2}^{(B)}$ utilizing the model of Eq. S13.

\section{S5 Spectral analysis}

The absorption spectrum of TIPS-Pn in solution was fit using a vibronic progression model: [5, 6]

$$
\frac{A(\hbar \omega)}{\hbar \omega}=A_{0} \sum_{m=0}^{4} \frac{e^{-S} S^{m}}{m !} \Gamma_{\sigma_{00}(1+m \Delta \sigma)}\left(\hbar \omega-E_{X}-m E_{V}\right)
$$

where $A / \hbar \omega$ is the reduced absorption, $A_{0}$ is a normalization constant, $S$ is the Huang-Rhys factor, $\Gamma_{\sigma}$ is a normalized Lorentzian lineshape with half width at half maximum (HWHM) of $\sigma$ which incorporates $\sigma_{00}$ (the HWHM of the 0-0 peak) and $\Delta \sigma$ (a progressive broadening term). [6] Finally, $E_{X}$ is the 0-0 exciton energy and $E_{V}$ is the vibrational energy quantum.

In particular, the reduced absorption spectra of bare films were modeled as:

$$
A_{r e d}(\hbar \omega)=-\frac{1}{\hbar \omega} \log _{10}\left(R_{c}\right)=\sum_{i} A_{i} \Gamma_{\sigma_{i}}\left(\hbar \omega-E_{X}^{(0 i)}\right)
$$

where $A_{r e d}$ is the reduced absorption, $\hbar \omega$ is the incident photon energy, $R_{c}$ is the reflectance corrected for the plasma frequency the silver substrate of the film, $A_{i}$ is the area of the $i^{t h}$ reduced absorbance resonance, $\Gamma_{\sigma_{i}}$ is a normalized Lorentzian (Gaussian) lineshape with HWHM (standard deviation) of $\sigma_{i}$ and $E_{X}^{(0 i)}$ is the resonance (exciton) energy.

In the case of films $F_{2}^{(B)}$ and $A^{(P)}$, in addition to the general model of Eq. (S17), the data were fit with the more specific vibronic progression (Eq. (S16)). Figures S5 and S8 show examples of the fits. To characterize the blends with contributions of amorphous and crystalline phases to the optical absorption (e.g. $F_{1.3}^{(B)}$ in Fig. S6), the absorption spectrum was fit to determine the relative contribution of these phases to the overall absorption. The amorphous phase contribution to the spectrum was modeled as a vibronic progression (Eq.(S16)) with the $E_{X}^{(00)}$ energy fixed to $1.91 \mathrm{eV}$, and the crystalline phase contribution was modeled using four Gaussian functions. Fit parameters are included in Table S2.

The angle resolved reflectance of the cavities was modeled using the following equation to extract the center energies of each feature: 


$$
R(\hbar \omega, \theta)=B S(\hbar \omega, \theta)+\sum_{n} C_{n}(\theta) \Gamma_{G_{n}(\theta)}\left(\hbar \omega-E_{n}(\theta)\right)
$$

where $R$ is the reflectance at photon energy $\hbar \omega$ and angle of incidence $\theta, B S$ is a baseline reflectance due to the silver substrate, and the resonances in the spectra are modeled with either a Lorentzian or Gaussian lineshape $(\Gamma)$ depending on the film morphology. $C_{n}(\theta)$ is the area, $G_{n}(\theta)$ is the half-width at the half maximum for Lorentzian lineshapes and the standard deviation for Gaussian lineshapes and $E_{n}(\theta)$ is the center energy for the $n^{\text {th }}$ resonance at angle of incidence $\theta$.

\section{S6 Optical absorption spectra with fits}

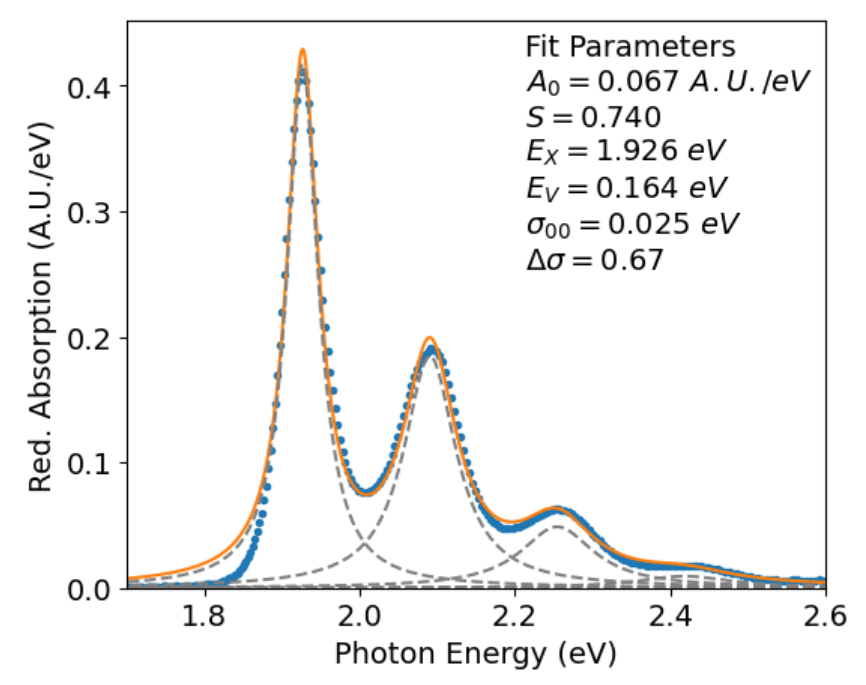

Figure S4: The reduced absorption spectrum for a $30 \mu M$ solution of TIPS-Pn in toluene. Experimental data is given as dots, and the fit according to a vibronic progression (Eq. (S16)) is given as a solid line; dashed lines show the deconvolved peaks. The inset gives the parameter values. 


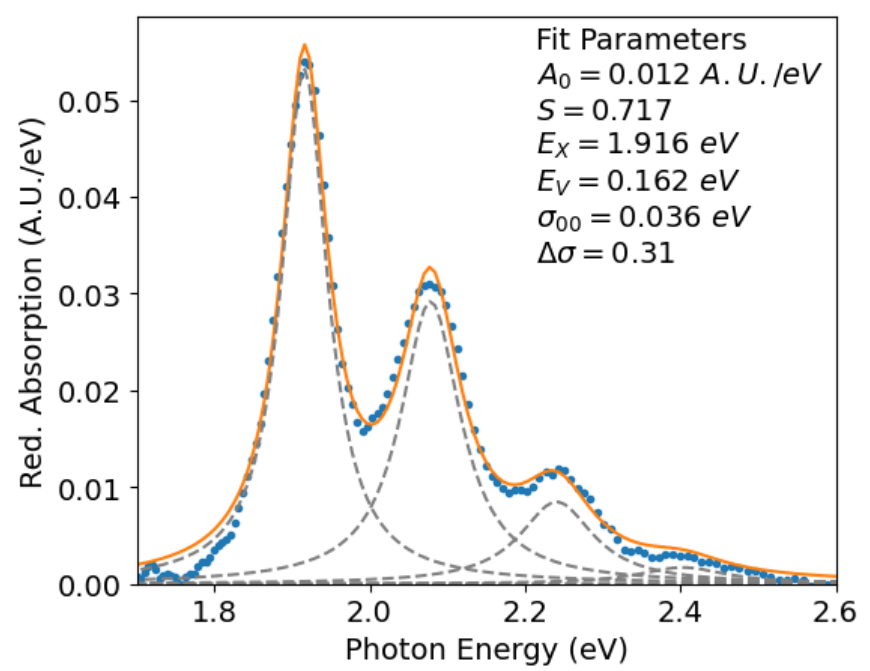

Figure S5: The reduced absorption spectrum for a film $F_{2}^{(B)}$. Experimental data is given as dots, and the fit according to a vibronic progression (Eq. (S16)) is given as a solid line; dashed lines show the deconvolved peaks. The inset gives the parameter values.

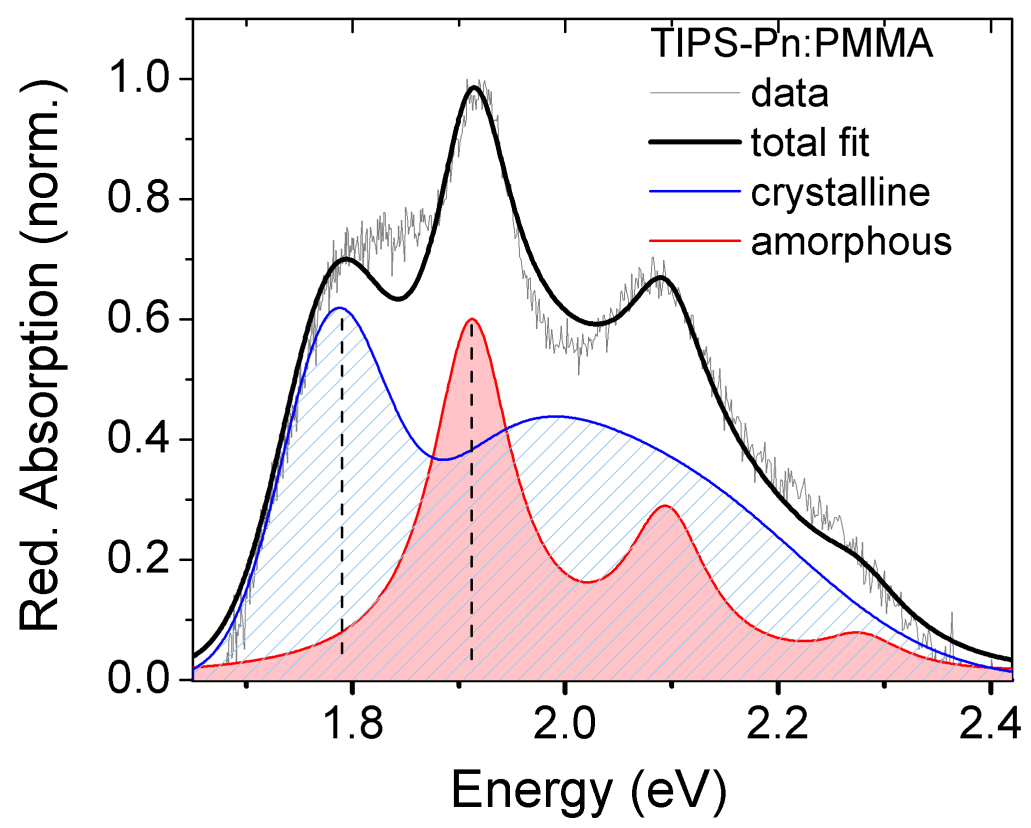

Figure S6: The reduced absorption spectrum for a film $F_{1.3}^{(B)}$. Contributions of the amorphous and crystalline phases (36\% and 64\%, respectively) obtained as described in Sec. S5 are indicated. Fit parameters are listed in Table S2. 


\begin{tabular}{|l|l|l|l|l|l|l|}
\hline Amorphous & $\mathrm{A}$ & $\mathrm{E}_{X^{*}}$ & $\mathrm{E}_{V}$ & $\mathrm{~S}$ & $\sigma_{00}$ & $\Delta \sigma$ \\
\hline & 0.128 & $1.912 \mathrm{eV}$ & $0.183 \mathrm{eV}$ & 0.43 & $93 \mathrm{meV}$ & $3.62 \cdot 10^{-7}$ \\
\hline
\end{tabular}

\begin{tabular}{|l|l|l|l|l|l|l|}
\hline Crystalline & $\mathrm{A}_{0}$ & $\mathrm{E}_{X}^{00} *$ & $\sigma_{0}$ & $\mathrm{~A}_{1}$ & $\mathrm{E}_{X}^{01} *$ & $\sigma_{1}$ \\
\hline & 0.522 & $1.78 \mathrm{eV}$ & $48 \mathrm{meV}$ & 0.268 & $1.93 \mathrm{eV}$ & $95 \mathrm{meV}$ \\
\hline \hline & $\mathrm{A}_{2}$ & $\mathrm{E}_{X}^{02}$ & $\sigma_{2}$ & $\mathrm{~A}_{3}$ & $\mathrm{E}_{X}^{03}$ & $\sigma_{3}$ \\
\hline & 0.316 & $2.1 \mathrm{eV}$ & $128 \mathrm{meV}$ & $4.98 \cdot 10^{-5}$ & $2.29 \mathrm{eV}$ & $45 \mathrm{meV}$ \\
\hline
\end{tabular}

Table S2: Fitting parameters for the film $F_{1.3}^{(B)}$ using the vibronic progression of Eq.(S16) for the amorphous part and model of Eq.(S17) with Gaussian lineshapes for the crystalline part. The asterisk "*" indicates the value was fixed during fitting. These values were informed by fits of $F_{2}^{(B)}$ for the amorphous part and $F_{1}^{(B)}$ and $F^{(P)}$ for the crystalline part.

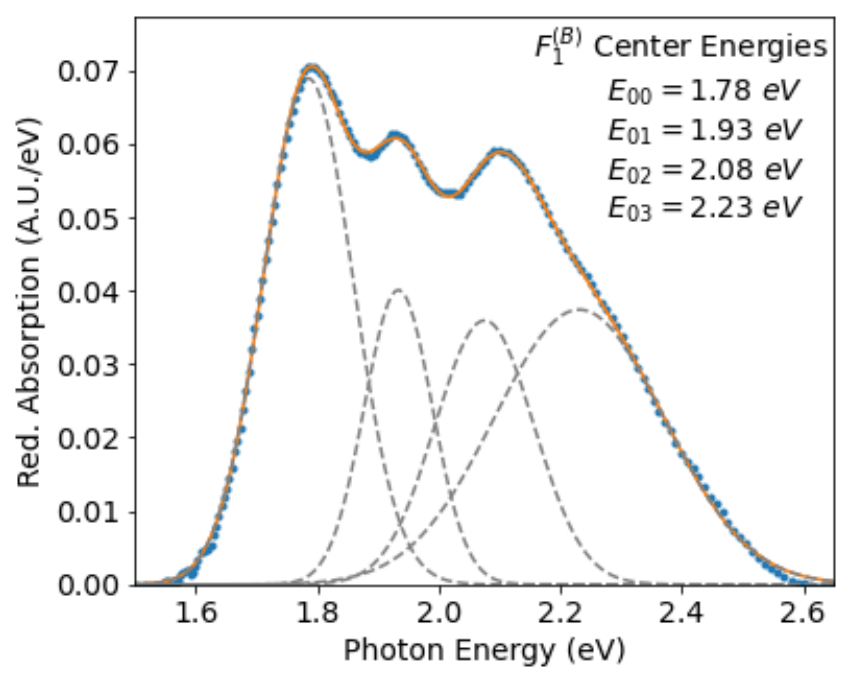

Figure S7: The reduced absorption spectrum for a film $F_{1}^{(B)}$. Experimental data is given as dots, and the fit according to a general model of Eq. (S17), with four Gaussian functions, is given as a solid line; dashed lines show the deconvolved peaks. The inset shows peak energies; the full set of fit parameter values is included in Table S3. Fits with a combination of the contributions due to the amorphous phase (characterized by a $\sim 1.9 \mathrm{eV} 0-0$ energy and vibronic progression of Eq. (S16)) and the crystalline phase (characterized by four Gaussian functions) were also performed, with varying contributions of the amorphous phase to the overall absorption, but did not result in the improved fit quality.

\begin{tabular}{|l|l|l|l|l|l|}
\hline $\mathrm{A}_{0}$ & $\mathrm{E}_{X}^{00}$ & $\sigma_{0}$ & $\mathrm{~A}_{1}$ & $\mathrm{E}_{X}^{01}$ & $\sigma_{1}$ \\
\hline 0.0689 & $1.785 \mathrm{eV}$ & $74 \mathrm{meV}$ & 0.0400 & $1.933 \mathrm{eV}$ & $56 \mathrm{meV}$ \\
\hline \hline $\mathrm{A}_{2}$ & $\mathrm{E}_{X}^{02}$ & $\sigma_{2}$ & $\mathrm{~A}_{3}$ & $\mathrm{E}_{X}^{03}$ & $\sigma_{3}$ \\
\hline 0.0359 & $2.075 \mathrm{eV}$ & $81 \mathrm{meV}$ & 0.0374 & $2.231 \mathrm{eV}$ & $134 \mathrm{meV}$ \\
\hline
\end{tabular}

Table S3: Fitting parameters for the film $F_{1}^{(B)}$ using the model of Eq.(S17) with Gaussian lineshapes. 


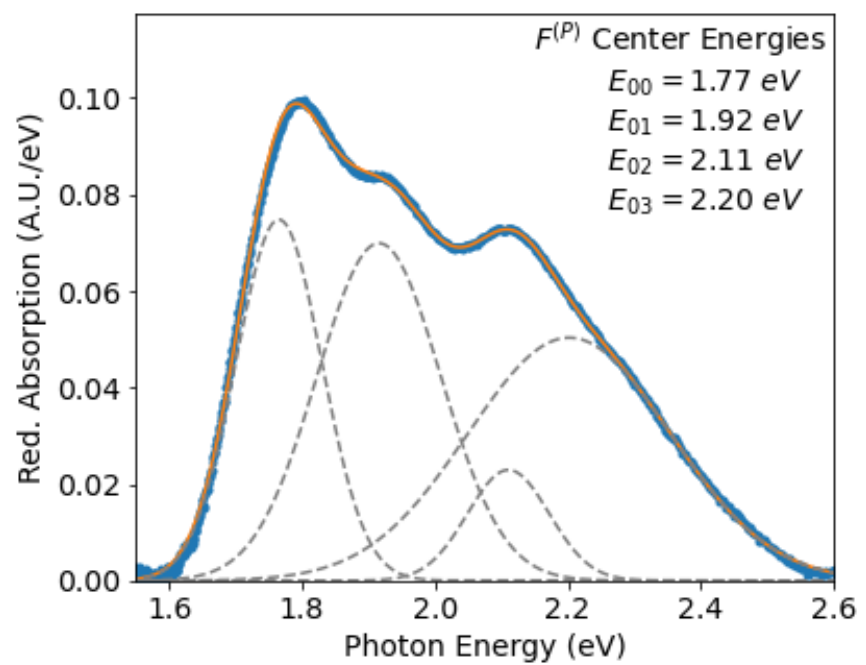

Figure S8: The reduced absorption spectrum for a film $F^{(P)}$. Experimental data is given as dots, and the fit with Eq. (S17) using Gaussian lineshapes is given as a solid line; dashed lines show the deconvolved peaks. The inset shows peak energies; the full set of fit parameter values is included in Table S4.

\begin{tabular}{|l|l|l|l|l|l|}
\hline $\mathrm{A}_{0}$ & $\mathrm{E}_{X}^{00}$ & $\sigma_{0}$ & $\mathrm{~A}_{1}$ & $\mathrm{E}_{X}^{01}$ & $\sigma_{1}$ \\
\hline 0.0748 & $1.765 \mathrm{eV}$ & $65 \mathrm{meV}$ & 0.0699 & $1.916 \mathrm{eV}$ & $93 \mathrm{meV}$ \\
\hline \hline $\mathrm{A}_{2}$ & $\mathrm{E}_{X}^{02}$ & $\sigma_{2}$ & $\mathrm{~A}_{3}$ & $\mathrm{E}_{X}^{03}$ & $\sigma_{3}$ \\
\hline 0.0229 & $2.110 \mathrm{eV}$ & $61 \mathrm{meV}$ & 0.0503 & $2.202 \mathrm{eV}$ & $152 \mathrm{meV}$ \\
\hline
\end{tabular}

Table S4: Fitting parameters for the film $F^{(P)}$ using the model of Eq.(S17) with Gaussian lineshapes.

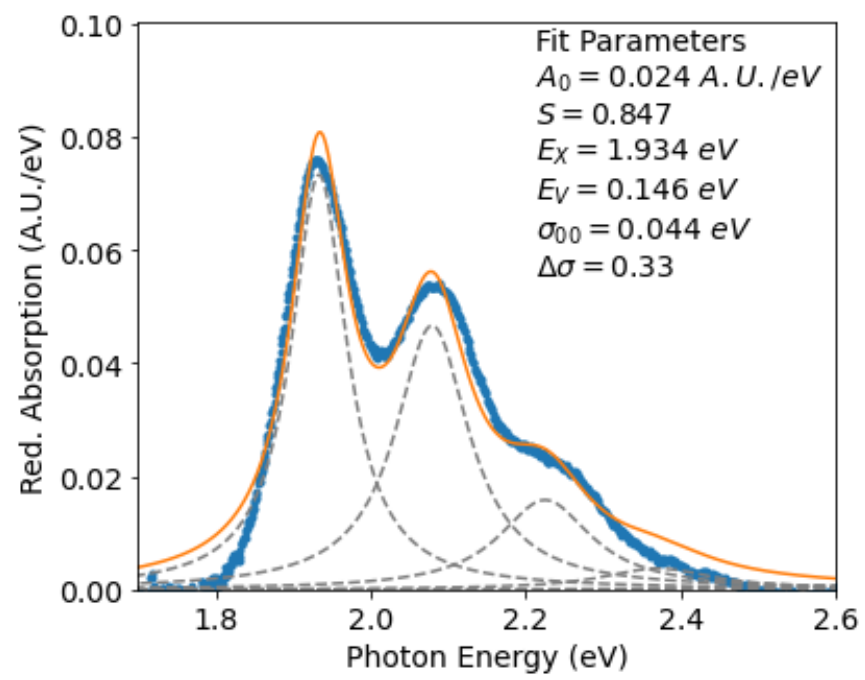

Figure S9: The reduced absorption spectrum for a film $A^{(P)}$. Experimental data is given as dots, and the fit according to a vibronic progression (Eq. (S16)) is given as a solid line; dashed lines show the deconvolved peaks. The inset gives the parameter values. 


\section{S7 Photoluminescence spectra and lifetimes}

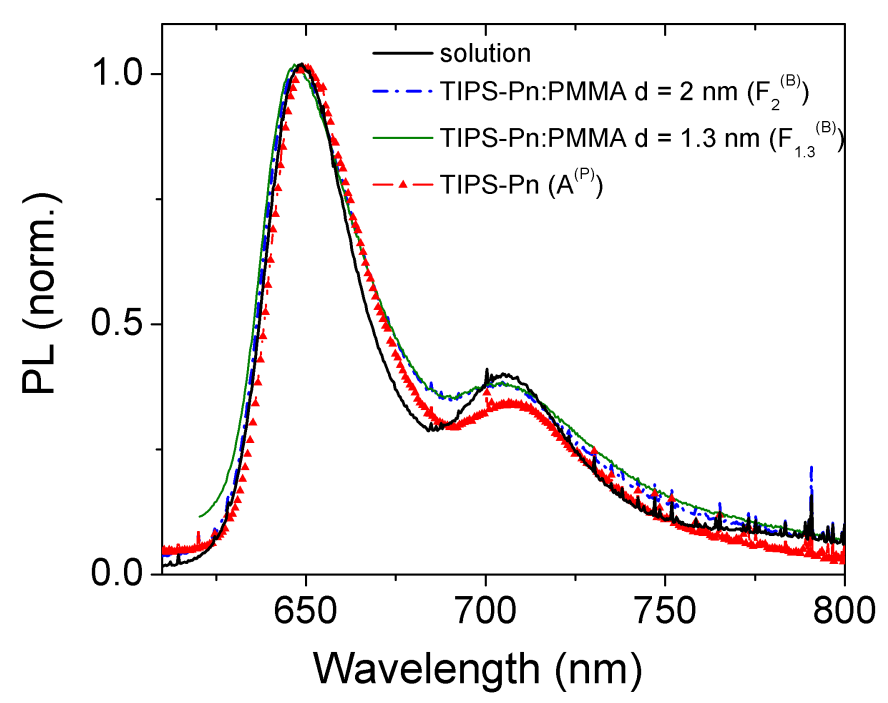

Figure S10: Normalized PL spectra of TIPS-Pn in toluene, of TIPS-Pn:PMMA blends $F_{2}^{(B)}$ and $F_{1.3}^{(B)}$, and amorphous pristine TIPS-Pn film $A^{(P)}$.

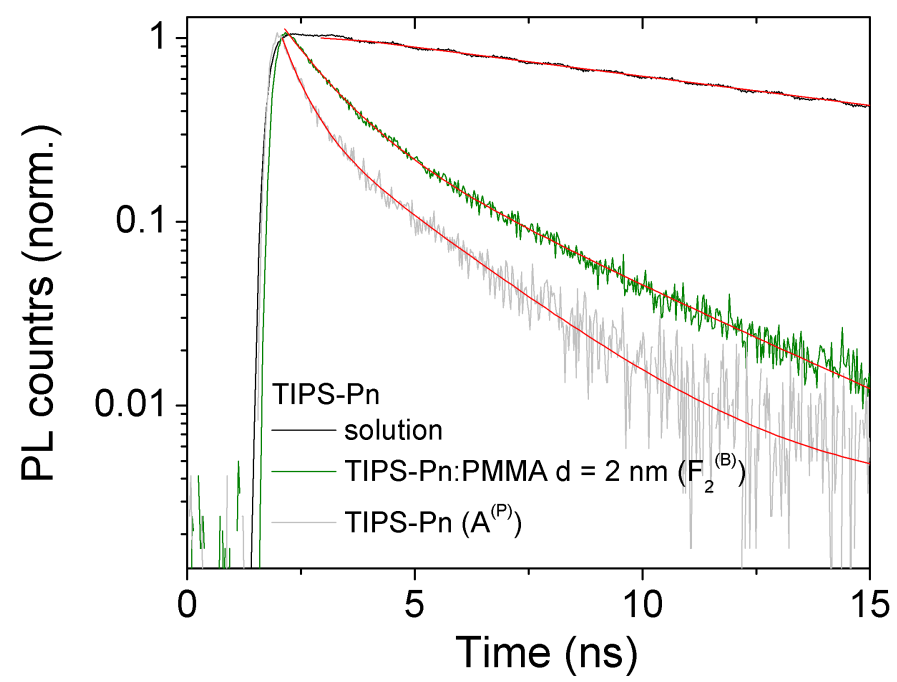

Figure S11: Time-resolved PL decays normalized by the counts at the maximum for TIPS-Pn in toluene, TIPS-Pn:PMMA films $F_{2}^{(B)}$ and $F_{1.3}^{(B)}$, and pristine amorphous film $A^{(P)}$. The fits to a monoexponential $A_{1} e^{-\left(t-t_{0}\right) / \tau_{1}}$ for the solution and a biexponential $A_{1} e^{-\left(t-t_{0}\right) / \tau_{1}}+A_{2} e^{-\left(t-t_{0}\right) / \tau_{2}}$ for the films are also shown (red lines). The lifetimes $\tau_{1}$ and $\tau_{2}$ and their weights are listed in Table S5 


\begin{tabular}{|l|l|l|l|l|}
\hline & $\tau_{1}(n s)$ & $\tau_{2}(n s)$ & $A_{1}$ & $A_{2}$ \\
\hline Solution & $13.76 \pm 0.02$ & - & 1 & - \\
\hline$F_{2}^{(B)}$ & $1.37 \pm 0.03$ & $5.2 \pm 0.5$ & 0.82 & 0.18 \\
\hline$A^{P}$ & $0.57 \pm 0.01$ & $2.98 \pm 0.07$ & 0.74 & 0.26 \\
\hline
\end{tabular}

Table S5: Fitting parameters for PL decay dynamics. The decays were fit to a monoexponential $A_{1} e^{-\left(t-t_{0}\right) / \tau_{1}}$ for the solution and a biexponential $A_{1} e^{-\left(t-t_{0}\right) / \tau_{1}}+A_{2} e^{-\left(t-t_{0}\right) / \tau_{2}}$ for the films.

\section{S8 Polariton properties}

The center energies of the cavity reflection resonances were modeled using a coupled oscillators model for polaritons [1]

$$
H=\left[\begin{array}{ccccc}
E_{p h}(\theta) & V_{00} & V_{01} & V_{02} & \cdots \\
V_{00} & E_{X}^{(00)} & 0 & 0 & \cdots \\
V_{01} & 0 & E_{X}^{(01)} & 0 & \ldots \\
V_{02} & 0 & 0 & E_{X}^{(02)} & \ldots \\
\vdots & \vdots & \vdots & \vdots & \ddots
\end{array}\right]
$$

where $E_{p h}(\theta)$ is the cavity photon energy at angle of incidence $\theta, E_{X}^{(0 i)}$ is the energy of the $i^{\text {th }}$ exciton and $V_{0 i}$ is the corresponding interaction energy. The number of excitons included in the matrix depends upon the film, see Table $\mathrm{S} 6$ for details. The cavity photon $E_{p h}$ was modeled with:

$$
E_{p h}(\theta)=E_{0}\left(1-\left(\frac{\sin (\theta)}{n_{\text {eff }}}\right)^{2}\right)^{-\frac{1}{2}}
$$

where $E_{0}$ is the cavity photon energy at normal incidence and $n_{\text {eff }}$ is the effective refractive index. The Hamiltonian was numerically diagonalized at each angle of incidence to obtain the polariton branch energies which were then compared to the center energies $E_{n}(\theta)$ in a nonlinear least squares optimization to determine the interaction strengths $V_{0 m}$. All fit parameters are given in Table $\mathrm{S} 6$. 


\begin{tabular}{|c|c|c|c|c|c|c|c|c|}
\hline \multirow[b]{2}{*}{ Film } & \multirow[b]{2}{*}{$\mathrm{RPM}$} & \multirow[b]{2}{*}{ Pol. } & \multirow[b]{2}{*}{$E_{p h}^{0}$} & \multirow[b]{2}{*}{$n_{e f f}$} & \multicolumn{4}{|c|}{$2 V_{0 i}\left(E_{X}^{0 i}\right)$} \\
\hline & & & & & 0 & 1 & 2 & 3 \\
\hline$F_{2}^{(B)}$ & 2000 & $\mathrm{~s}$ & $1.812 \mathrm{eV}$ & 1.530 & $\begin{array}{c}85 \mathrm{meV} \\
(1.91 \mathrm{eV})\end{array}$ & $\begin{array}{c}62 \mathrm{meV} \\
(2.09 \mathrm{eV})\end{array}$ & $\begin{array}{c}16 \mathrm{meV} \\
(2.23 \mathrm{eV})\end{array}$ & $\sim$ \\
\hline$F_{2}^{(B)}$ & 2000 & $\mathrm{p}$ & $1.813 \mathrm{eV}$ & 2.072 & $\begin{array}{c}86 \mathrm{meV} \\
(1.91 \mathrm{eV})\end{array}$ & $\begin{array}{c}66 \mathrm{meV} \\
(2.08 \mathrm{eV})\end{array}$ & $\sim$ & $\sim$ \\
\hline$F_{1}^{(B)}$ & 2500 & $\mathrm{~s}$ & $1.732 \mathrm{eV}$ & 1.685 & $\begin{array}{l}228 \mathrm{meV} \\
(1.91 \mathrm{eV})\end{array}$ & $\begin{array}{l}182 \mathrm{meV} \\
(2.07 \mathrm{eV})\end{array}$ & $\begin{array}{l}121 \mathrm{meV} \\
(2.24 \mathrm{eV})\end{array}$ & $\sim$ \\
\hline$F_{1}^{(B)}$ & 2500 & $\mathrm{p}$ & $1.721 \mathrm{eV}$ & 2.194 & $\begin{array}{l}207 \mathrm{meV} \\
(1.91 \mathrm{eV})\end{array}$ & $\begin{array}{l}166 \mathrm{meV} \\
(2.07 \mathrm{eV})\end{array}$ & $\sim$ & $\sim$ \\
\hline$F^{(P)}$ & 1000 & $\mathrm{~s}$ & $1.802 \mathrm{eV}$ & 1.893 & $\begin{array}{l}210 \mathrm{meV} \\
(1.73 \mathrm{eV})\end{array}$ & $\begin{array}{l}215 \mathrm{meV} \\
(1.93 \mathrm{eV})\end{array}$ & $\begin{array}{l}190 \mathrm{meV} \\
(2.12 \mathrm{eV})\end{array}$ & $\begin{array}{l}225 \mathrm{meV} \\
(2.29 \mathrm{eV})\end{array}$ \\
\hline$F^{(P)}$ & 1000 & $\mathrm{p}$ & $1.766 \mathrm{eV}$ & 2.102 & $\begin{array}{l}172 \mathrm{meV} \\
(1.73 \mathrm{eV})\end{array}$ & $\begin{array}{l}217 \mathrm{meV} \\
(1.93 \mathrm{eV})\end{array}$ & $\begin{array}{l}229 \mathrm{meV} \\
(2.12 \mathrm{eV})\end{array}$ & $\begin{array}{l}232 \mathrm{meV} \\
(2.29 \mathrm{eV})\end{array}$ \\
\hline$A^{(P)}$ & 3000 & $\mathrm{~s}$ & $1.832 \mathrm{eV}$ & 1.752 & $\begin{array}{l}253 \mathrm{meV} \\
(1.93 \mathrm{eV})\end{array}$ & $\begin{array}{l}276 \mathrm{meV} \\
(2.09 \mathrm{eV})\end{array}$ & $\begin{array}{l}246 \mathrm{meV} \\
(2.25 \mathrm{eV})\end{array}$ & $\sim$ \\
\hline$A^{(P)}$ & 3000 & $\mathrm{p}$ & $1.851 \mathrm{eV}$ & 2.306 & $\begin{array}{l}248 \mathrm{meV} \\
(1.93 \mathrm{eV})\end{array}$ & $\begin{array}{l}236 \mathrm{meV} \\
(2.09 \mathrm{eV})\end{array}$ & $\sim$ & $\sim$ \\
\hline$A^{(P)}$ & 3500 & $\mathrm{~s}$ & $1.953 \mathrm{eV}$ & 1.837 & $\begin{array}{l}229 \mathrm{meV} \\
(1.93 \mathrm{eV})\end{array}$ & $\begin{array}{l}234 \mathrm{meV} \\
(2.09 \mathrm{eV})\end{array}$ & $\begin{array}{l}168 \mathrm{meV} \\
(2.25 \mathrm{eV})\end{array}$ & $\sim$ \\
\hline$A^{(P)}$ & 3500 & $\mathrm{p}$ & $1.934 \mathrm{eV}$ & 2.632 & $\begin{array}{l}214 \mathrm{meV} \\
(1.93 \mathrm{eV})\end{array}$ & $\begin{array}{l}204 \mathrm{meV} \\
(2.09 \mathrm{eV})\end{array}$ & $\begin{array}{l}166 \mathrm{meV} \\
(2.25 \mathrm{eV})\end{array}$ & $\sim$ \\
\hline
\end{tabular}

Table S6: The polariton properties for representative cavities containing each of the four films: $F_{2}^{(B)}, F_{1}^{(B)}, F^{(P)}, A^{(P)}$, indexed by the spin-casting RPM for the associated film. The table lists the incident polarization of light (Pol.), the normal-incidence photon energy $\left(E_{p h}^{0}\right)$ and the effective index of refraction for that polarization $\left(n_{\text {eff }}\right)$ used in Eq. (5) in the main text. Cited also is the exciton energy for the $0-i$ absorptive transition $(i=0,1,2,3)$ as well as the interaction strength $\left(2 V_{0 i}\right)$ associated with that exciton according to Eq. (S19). In the case of amorphous films $\left(F_{2}^{(B)}, F_{1}^{(B)}\right.$, and $\left.A^{(P)}\right)$, only the first three excitons had sufficient oscillator strength to resolvably couple to the cavity photon, so $i=4$ is not included. Additionally, for ppolarized incident light with a red-detuned cavity containing amorphous film $A^{(P)}$, occasionally the photon at high angles of incidence was not high enough in energies to mix efficiently with the third exciton $(i=2)$, and therefore accurate estimation of the interaction strength in these cases was not possible, and so these values are omitted. 


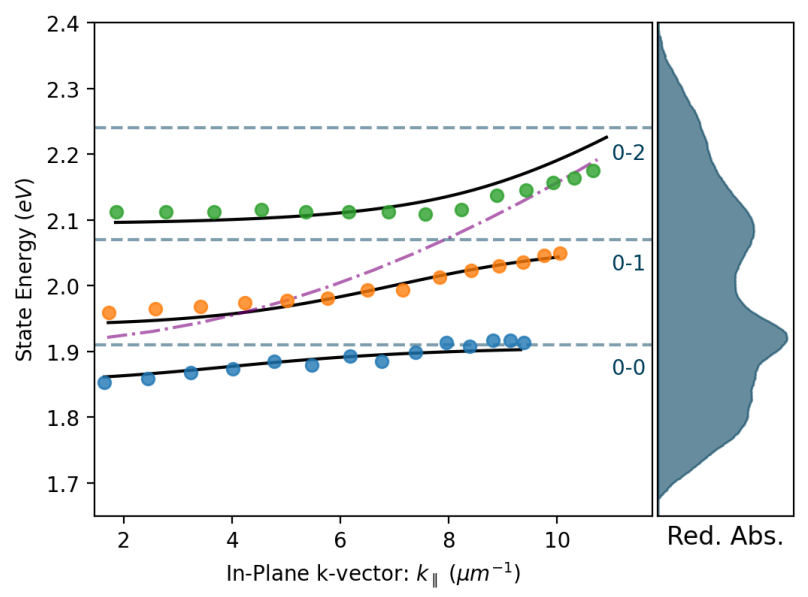

Figure S12: A dispersion plot of the polariton energies extracted from the reflectance from mixed-phase blend $F_{1.3}^{(B)}$. Dots show the experimentally extracted energies, the dash-dotted line shows the photon energy, the dashed line shows the exciton energies and solid black lines show fits according to the coupled oscillator model. Shown on the right is the absorption spectrum of the bare film to illustrate that the cavity photon couples to the exciton in the amorphous phase (lowest-energy exciton at $\sim 1.93 \mathrm{eV}$ ) rather than that in the crystalline phase (lowest-energy exciton at $\sim 1.78 \mathrm{eV})$. 

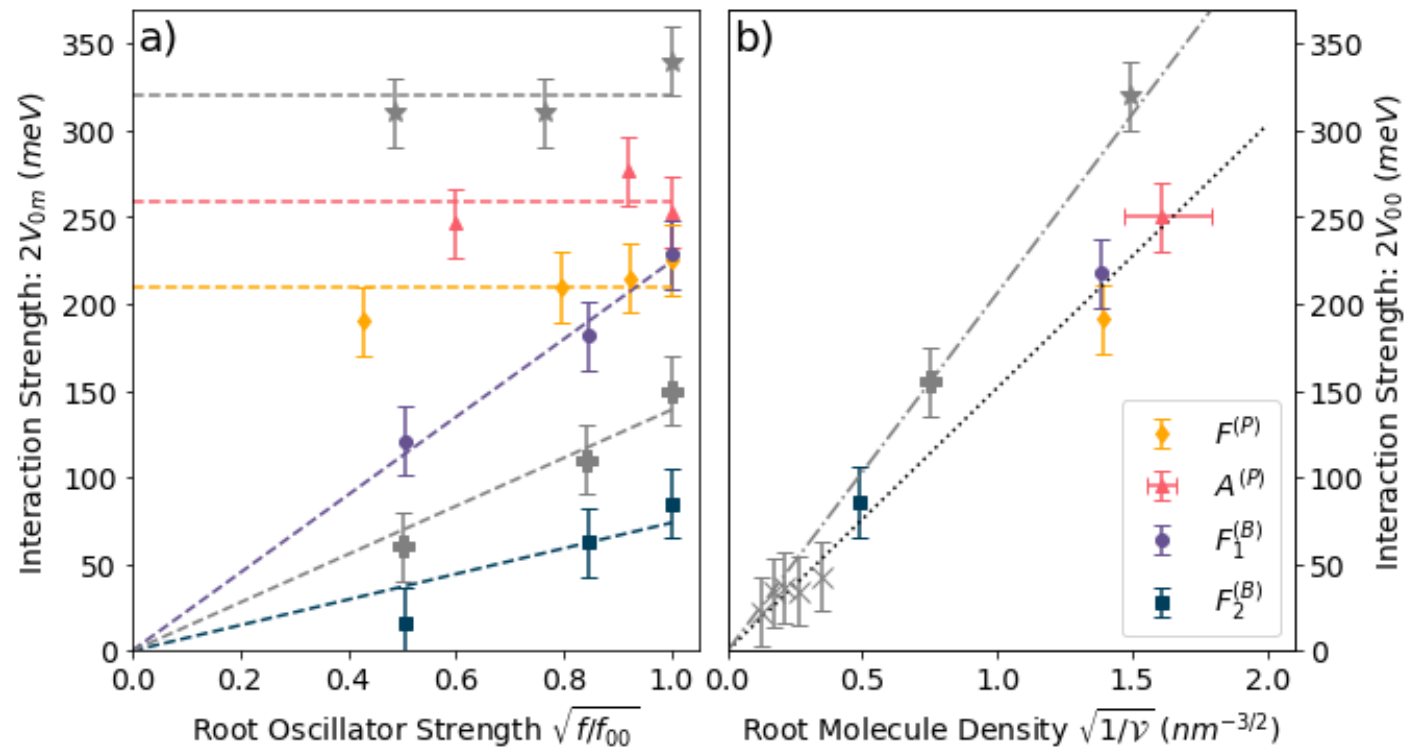

Figure S13: (a) A comparison of the interaction strengths $\left(2 V_{0 m}, m=0,1,2,3\right)$ for s-polarized polaritons in each cavity $\left(F_{2}^{(B)}, F_{1}^{(B)}, F^{(P)}\right.$, and $\left.A^{(P)}\right)$ as plotted against the square root of the oscillator strength (relative to that of the 0-0 exciton) for the associated exciton (see caption of Fig. 6(a) in the main text for details). The dashed lines for each set of data represent either a proportional fit or constant fit (Eq. (2) in the main text) to highlight the deviation from a proportional model. The "star" and "plus" markers show comparable data for diF TES-ADT:PMMA blends for crystalline phase (in fully crystalline $P_{\text {agg }}$ films with optical absorption spectra characteristic of a "crystalline" phase) and amorphous phase (in $P_{1.5}$ films with an average diF TES-ADT spacing of $1.5 \mathrm{~nm}$, with contributions from both crystalline and amorphous phases to the optical absorption spectra), respectively.[1] (b) A comparison of the 0-0 interaction strength $\left(2 V_{00}\right)$, averaged for s- and p-polarized polaritons in each cavity type, plotted against the square root of the effective molecular density $N_{\text {tot }}=1 / \mathcal{V}$ where $\mathrm{V}$ is the molecular volume (see caption of Fig. 6(b) in the main text for details). The dotted line represents a proportional fit, based on Eq. (3) in the main text. Vertical error bars were estimated at $20 \mathrm{meV}$ based of the coupled oscillators fit. The "star", "plus" and "x" markers show comparable data for the 0-0 exciton interaction strength for diF TES-ADT:PMMA in crystalline phase of film $P_{a g g}$ ("star") and amorphous phases in films $P_{1.5}$ ("plus"), and $P_{d}$ with d ranging between $2.5 \mathrm{~nm}$ and $6 \mathrm{~nm}$ ("x"). [1] 

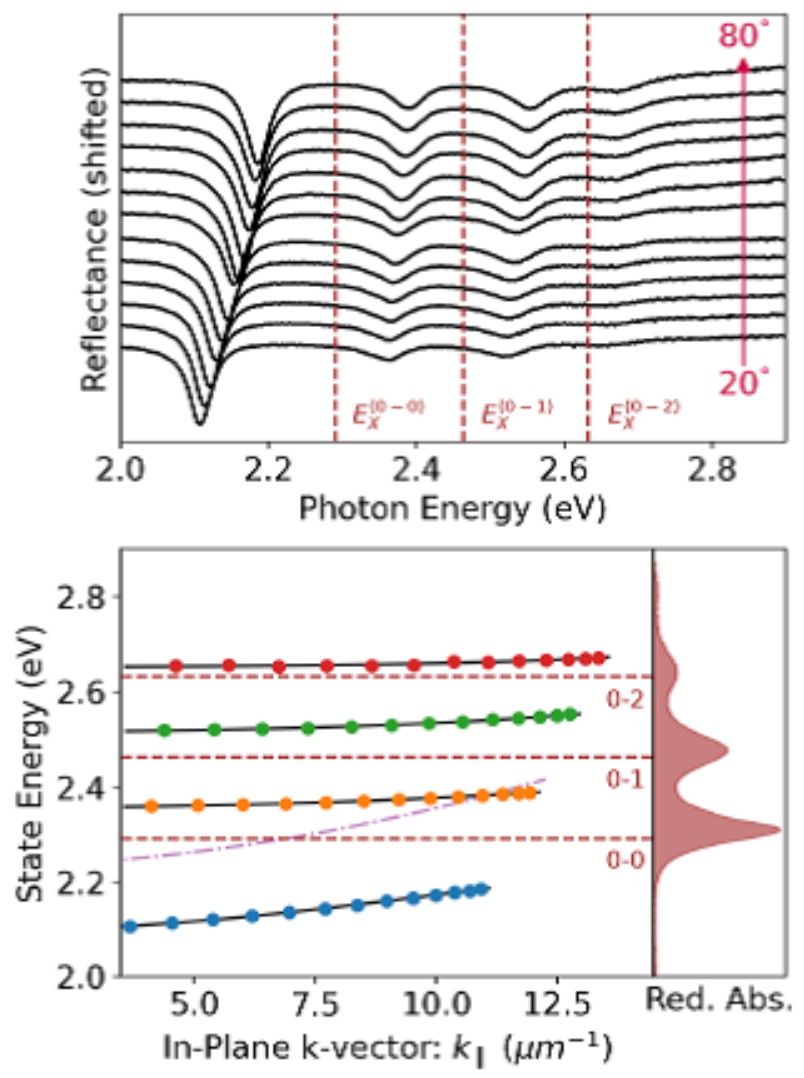

Figure S14: (a) Angle-dependent reflectance spectra for amorphous TIPS-Tc film in an allmetal cavity for s-polarization. Vertical dashed lines label exciton resonances in TIPS-Tc corresponding to 0-0, 0-1, and 0-2 transitions. (b) Dispersion characteristics obtained from reflectance dip positions in the data in (a) (dots) fit with the coupled oscillators model (lines) to determine coupling strengths $\left(2 V_{00}=266 \mathrm{meV}, 2 \mathrm{~V}_{01}=227 \mathrm{meV}, 2 \mathrm{~V}_{02}=140 \mathrm{meV}\right)$. Lower polariton (LP) (blue dots), middle polariton 1 (MP1) (orange dots), middle polariton 2 MP2) (green dots), and upper polariton (UP) (red dots) branches are shown. Dash-dotted line corresponds to the dispersion of the cavity photon, and horizontal dashed lines show the energies of the excitons in bare (uncoupled to the cavity) TIPS-Tc films for which the absorption spectrum is included on the right. Data are taken from Ref.[7]. 


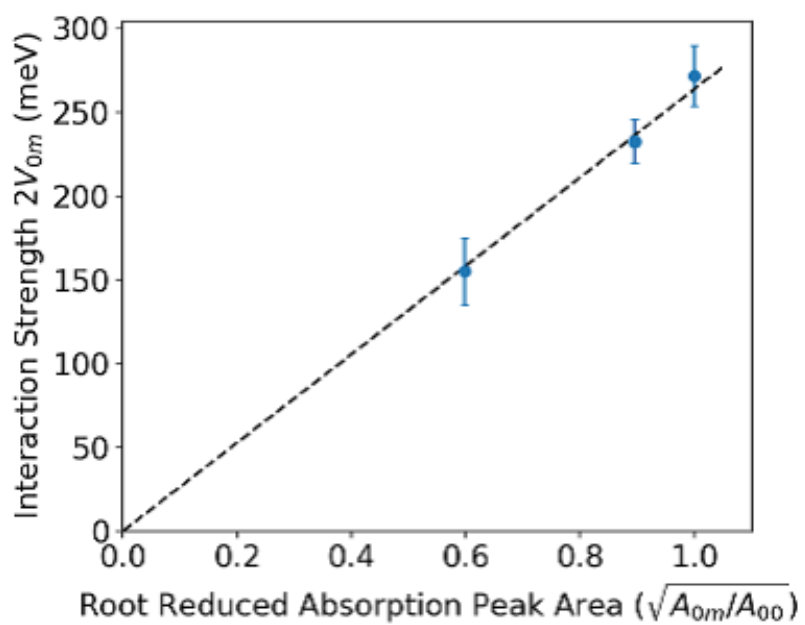

Figure S15: Linear scaling of the coupling strength $2 V_{0 m}$ with the square root of the oscillator strength $f_{0 m}$ for exciton resonances $0-m(m=0,1,2)$ obtained from fits of the data to the model of Eq. (2) of the main text. Data are taken from Ref. [7.

\begin{tabular}{cc|ccc} 
Molecule & Film & Intercept $[\mathrm{meV}]$ & Slope $[\mathrm{meV}]$ & $\chi^{2} / \mathrm{df}$ \\
\hline \multirow{2}{*}{ TIPS-Pn } & $F_{2}^{(B)}$ & $0^{*}$ & $73 \pm 10$ & 0.79 \\
& $F_{1}^{(B)}$ & $0^{*}$ & $222 \pm 10$ & 0.18 \\
& $F^{(P)}$ & $210 \pm 7$ & $0^{*}$ & 0.55 \\
& $A^{(P)}$ & $258 \pm 8$ & $0^{*}$ & 0.63 \\
\multirow{2}{*}{$\operatorname{diF}$ TES-ADT } & $P_{1.5}$ & $0^{*}$ & $140 \pm 10$ & 0.0 \\
& $P_{\text {agg }}$ & $320 \pm 8$ & $0^{*}$ & 0.0 \\
\hline
\end{tabular}

Table S7: Fit parameters for Fig. 6(a) (Fig. S13(a)) in the main text (SI), "** indicates that the value was fixed during fitting. "†" indicates that the values are taken from Ref. [1]

\begin{tabular}{c|cc} 
Molecule & slope $\left[\mathrm{meV} / \mathrm{nm}^{-3 / 2}\right]$ & $\chi^{2} / \mathrm{df}$ \\
\hline TIPS-Pn & $166 \pm 8$ & 0.03 \\
diF TES-ADT $^{\dagger}$ & $239 \pm 9$ & 1.13 \\
\hline
\end{tabular}

Table S8: Fit parameters for Fig. 6(b) (Fig. S13(b)) in the main text (SI). "†" indicates that the values are taken from Ref. [1]

\section{S9 Density functional theory}

The molecular configuration of $\mathrm{SiH}_{3}-\mathrm{Pn}, \mathrm{SiH}_{3}-\mathrm{Tc}$, and $\mathrm{diF} \mathrm{SiH}_{3}-\mathrm{ADT}$ molecules was optimized via Density Functional Theory (DFT) methods using the Gaussian 16 software suite. An initial geometry was optimized using the B3LYP functional and the $6-311+\mathrm{G}(2 \mathrm{~d}, \mathrm{p})$ basis set. The excited states were then calculated using Time-dependent DFT (TD-DFT) as described in our previous publications. [5, 7] The energies of the first three electronic transitions for each molecule and the corresponding oscillator strengths are summarized in Table S9. 


\begin{tabular}{cc|ccc} 
Molecule & Symmetry & $E_{X}^{\left(S_{0}-S_{1}\right)}\left(f^{\left(S_{0}-S_{1}\right)}\right)$ & $E_{X}^{\left(S_{0}-S_{2}\right)}\left(f^{\left(S_{0}-S_{2}\right)}\right)$ & $E_{X}^{\left(S_{0}-S_{3}\right)}\left(f^{\left(S_{0}-S_{3}\right)}\right)$ \\
\hline \multirow{2}{*}{$\mathrm{diF} \mathrm{SiH}_{3}-\mathrm{ADT}$} & $\mathrm{C} 2 \mathrm{~h}$ & $2.183 \mathrm{eV}(0.1997)$ & $3.110 \mathrm{eV}(0.0)$ & $3.197 \mathrm{eV}(0.0272)$ \\
& $\mathrm{Cs}$ & $2.185 \mathrm{eV}(0.1905)$ & $3.059 \mathrm{eV}(0.03)$ & $3.262 \mathrm{eV}(0.0)$ \\
$\mathrm{SiH}_{3}-\mathrm{Pn}$ & $\mathrm{C} 2 \mathrm{~h}$ & $1.654 \mathrm{eV}(0.1341)$ & $2.662 \mathrm{eV}(0.0)$ & $3.105 \mathrm{eV}(0.0309)$ \\
& $\mathrm{C} 2 \mathrm{v}$ & $1.655 \mathrm{eV}(0.1341)$ & $2.662 \mathrm{eV}(0.0)$ & $3.105 \mathrm{eV}(0.0305)$ \\
$\mathrm{SiH}_{3}-\mathrm{Tc}$ & - & $2.987 \mathrm{eV}(0.1663)$ & $3.117 \mathrm{eV}(0.0486)$ & - \\
\hline
\end{tabular}

Table S9: The energies of the first three electronic transitions for each molecule, depending on the symmetry (e.g. $C_{2 h}$ for trans-ADT and $C_{s}$ for syn-ADT). [5] The corresponding oscillator strengths are shown in parenthesis. For $\mathrm{SiH}_{3}-\mathrm{Tc}$, because the side groups are asymmetrically attached to the Tc core, no specific molecular symmetry was enforced during any DFT calculations.

The TD-DFT-calculated oscillator strength $(f)$ of 0.13 for the $S_{0}-S_{1}$ transition in $\mathrm{SiH}_{3}-\mathrm{Pn}$ was used to estimate the transition dipole moment $\mu$ as follows: 8$] \mu=e \sqrt{f /\left(l_{0} \nu\right)}$, where $e$ is the electric charge, $\nu$ is the wavenumber for the $S_{0}-S_{1}$ transition (taken to be $15,500 \mathrm{~cm}^{-1}$ ), and $l_{0}=1.085 \times 10^{-5} \mathrm{~cm} / \AA^{2}$, which yields $\mu=4.2 \mathrm{D}$. (Similar value of $3.05 \mathrm{D}$ for the first excited state transition was obtained in bitetracene in Ref. 9.

\section{References}

[1] J. Van Schenck, E. K. Tanyi, L.-J. Cheng, J. Anthony and O. Ostroverkhova "Strong exciton-photon coupling in anthradithiophene microcavities: from isolated molecules to aggregates" MRS Communications (2019) 9:3, 956-963

[2] W. Shepherd, A. Platt, D. Hofer, O. Ostroverkhova, M. Loth, J. Anthony, "Aggregate formation and its effect on (opto)electronic properties of guest-host organic semiconductors" Applied Physics Letters (2010) 97, 163303

[3] J. E. Anthony, J. S. Brooks, D. L. Eaton, and S. R. Parkin "Functionalized pentacene: improved electronic properties from control of solid-state order" Journal of the American Chemical Society (2001) 123(38), 9482-9483

[4] K. T. Munson, J. Gan, C. Grieco, G. S. Doucette, J. E. Anthony, and J. B. Asbury "Ultrafast triplet pair separation and triplet trapping following singlet fission in amorphous pentacene Films" Journal of Physical Chemistry C (2020) 124(43), 23567-23578

[5] J. Van Schenck, G. Mayonado, J. Anthony, M Graham, and O. Ostroverkhova, "Molecular packing-dependent exciton dynamics in functionalized anthradithiophene derivatives: from solutions to crystals" Journal of Chemical Physics (2020) 153, 164715

[6] H. Yamagata, D. Maxwell, J. Fan, K. Kittilstved, A. Briseno, M. Barnes, F. C. Spano, Journal of Physical Chemistry C (2014) 118, 28842

[7] R. Puro, J. D. B. Van Schenck, E. Holland, J. E. Anthony, O. Ostroverkhova "Exciton polariton-enhanced photodimerization of functionalized tetracene" Journal of Physical Chemistry $C$ (2021) accepted

[8] G. Calzaferri and R. Rytz "Electronic Transition Oscillator Strength by the Extended Hueckel Molecular Orbital Method" Journal of Chemical Physics (1995) 99, 12141-12150

[9] K. Shizu, C. Adachi, H. Kaji "Correlated Triplet Pair Formation Activated by Geometry Relaxation in Directly Linked Tetracene Dimer (5,5-Bitetracene)" ACS Omega (2021) 6, 2638-2643 